\title{
Effects of Antihypertensive Drugs on Thyroid Function in Type 2 Diabetes Patients With Euthyroidism
}

\author{
Lijuan Yang, Xiuqin Sun, Yi Zhao and Hong Tao* \\ Department of Endocrinology and Metabolism, Beijing Anzhen Hospital, Capital Medical University, Beijing, China
}

\section{OPEN ACCESS}

Edited by: Cristoforo Pomara,

University of Catania, Italy

Reviewed by:

Juan Dízz,

Hospital Universitario Puerta de Hierro

Majadahonda, Spain

Johannes Wolfgang Dietrich,

Ruhr University Bochum, Germany

*Correspondence:

Hong Tao

taohongthng@163.com

Specialty section:

This article was submitted to

Experimental Pharmacology and Drug

Discovery,

a section of the journal

Frontiers in Pharmacology

Received: 28 October 2021 Accepted: 04 January 2022

Published: 07 March 2022

Citation:

Yang $L$, Sun $X$, Zhao $Y$ and Tao $H$ (2022) Effects of Antihypertensive Drugs on Thyroid Function in Type 2 Diabetes Patients With Euthyroidism.

Front. Pharmacol. 13:802159.

doi: 10.3389/fphar.2022.802159
Objective: There is little literature about whether antihypertensive drugs would affect thyroid function in patients with euthyroid type 2 diabetes, which was significant in maintaining a proper balance of thyroid function. A retrospective cohort study was conducted to evaluate the influence of antihypertensive drugs on thyroid function in patients with type 2 diabetes with euthyroidism.

Design and Methods: The study involved dividing 698 patients with antihypertensive monotherapy into five groups according to the antihypertensive drugs they were treated with. Antihypertensive drugs included in this study were $\beta$-blockers, angiotensinconverting enzyme inhibitors (ACEI), angiotensin receptor blockers (ARB), and calcium channel blockers (CCB). The clinical data and thyroid function level between or within groups were compared. Multiple logistic regression analysis was conducted to evaluate the association of antihypertensive drugs with thyroid function level.

Results: Selective $\beta_{1}$-adrenergic receptor blockers treatment was related to thyroidstimulating hormone (TSH), increasing in patients with diabetes and euthyroidism as shown by multiple logistic regression analysis. The association existed after adjustment for confounding factors. No significant influence on thyroid function was found among other antihypertensive drugs.

Conclusion: These data show the TSH-lifting effect of selective $\beta_{1}$-adrenergic receptor blockers in patients with type 2 diabetes with euthyroidism.

Keywords: thyroid function, type 2 diabetes, thyroid-stimulating hormone, antihypertensive drugs, selective $\beta 1$ adrenergic receptor blockers

\section{INTRODUCTION}

Subjects with type 2 diabetes experience an elevated cardiovascular morbidity and mortality at present, associated with a high prevalence of hypertension, obesity, and dyslipidemia (Punthakee et al., 2007; Zhang et al., 2009). On the other hand, thyroid hormones play a crucial role in regulating many cardiovascular functions (Klein and Ojamaa, 2001). Previous literatures have revealed that decreased endothelial function, high-density lipoprotein cholesterol (HDL-C) level, and increased low-density lipoprotein cholesterol (LDL-C) level, triglycerides (TGs) level, and blood pressure (BP) were observed in persons with higher thyroid-stimulating hormone (TSH) level even when TSH level was still in reference range (Lekakis et al., 1997; Bakker et al., 2001; Asvold et al., 2007a; Asvold et al., 2007b). Therefore, accurately evaluating thyroid function in patients with type 2 diabetes, even when 
thyroid function was still in normal level, to keep a proper balance of thyroid hormones is necessary (Mullur et al., 2014).

Antidiabetic and antihypertensive treatments are both necessary in diabetes patients with hypertension. As we know, various drugs could influence thyroid function through different mechanisms (Andjelkovic et al., 2016). When evaluating thyroid function, the influence of medication should not be ignored. However, literatures about whether antihypertensive drugs would affect thyroid function were limited, especially in persons with euthyroidism.

Antihypertensive drugs used widely in clinical practice include $\beta$-blockers, angiotensin-converting enzyme inhibitors (ACEI), angiotensin receptor blockers (ARB), and calcium channel blockers (CCB) in our hospital. Evidence has linked propranolol therapy to a thyroid function alteration in patients with hyperthyroidism (Faber et al., 1979; Perret et al., 1984; Franklyn et al., 1985) and euthyroidism (Wilkins et al., 1985; Kayser et al., 1991). In recent years, selective $\beta_{1}$-adrenergic receptor blockers (second-generation agents) and thirdgeneration agents (Rizos et al., 2003; Bakris et al., 2004; Celik et al., 2006) are much more prevalent in clinical practice. However, the evidence for thyroid function-changing effect of second- and third-generation $\beta_{1}$-adrenergic receptor blockers in euthyroid cases shows contrary outcomes (Kristensen and Weeke, 1977; Faber et al., 1979; Perret et al., 1984; Reeves et al., 1985; Kayser et al., 1991). The effect of ACEI, ARB (Hume et al., 1990; Vranckx et al., 1995; Andjelkovic et al., 2016) and CCB (Brabant et al., 1989; Jangid, 1993; Eidne et al., 1994; Roussel et al., 1995; Roussel et al., 1997; Pedemonte et al., 2005) on thyroid function were not consistent between animal study and clinical studies and the sample size was small (Isles et al., 1985; Haitas et al., 1986; Valensi et al., 1989; Andjelkovic et al., 2016).

Therefore, a clinical study with a larger sample size might be required to investigate whether antihypertensive drugs would affect thyroid function in patients with type 2 diabetes when thyroid function was still in reference range.

\section{MATERIALS AND METHODS}

\section{Study Participants}

This was a retrospective cohort study examining the effect of antihypertensive therapy on thyroid function in patients with type 2 diabetes and euthyroidism. Patients with diabetes were recruited by searching the computerized database of patients who were treated and followed up at the Department of Endocrinology and Metabolism, Beijing Anzhen Hospital, from December 2014 to December 2020. There were 6,847 type 2 diabetes participants who had at least two visits at the baseline and the end during the 1-year follow-up were included to assess for eligibility. We excluded subjects with the following criteria: 1) without complete personal medical history and detailed drug treatment, missing vital data; 2) treatment with more than one antihypertensive drug during the 1-year follow-up, did not adhere to medication well; 3) thyroid function not within the normal range at baseline, thyroid disease history or therapy; 4) the usage of drugs known to affect thyroid function (metformin, glucocorticoids, dopamine agonists, rexinoids, carbamazepine, metyrapone, etc.) (Cappelli et al., 2012; Andjelkovic et al., 2016); 5) a history of serious cardiovascular events (ischemic heart disease, heart failure, etc.), stroke, chronic obstructive peripheral arteriopathy, gastrointestinal tract disorders (chronic gastritis, Helicobacter pylori infection, chronic pancreatitis, and inflammatory bowel disease, etc.), anemia or hemoglobinopathies, malignant disease, liver or renal dysfunction, and a recent history of acute illness; 6) pregnancy; and 7) drug or alcohol abuse.

Type 2 diabetes was diagnosed under World Health Organization (WHO) 1999 criteria (Alberti and Zimmet, 1999), or with a history of type 2 diabetes. Hypertension was defined as systolic blood pressure (SBP) of $\geq 140 \mathrm{mmHg}$ and/or diastolic blood pressure (DBP) of $\geq 90 \mathrm{mmHg}$ (Writing Group of Chinese Guidelines for the Management of Hypertension, 2010), or having a history of hypertension. Complications of diabetes and hypertension were recorded as retinopathy complication, kidney disease complication, and peripheral neuropathy complication. Retinopathy complication was determined by fundus examination. Kidney disease complication and peripheral neuropathy complication was defined according to the criteria of Chinese guidelines for the management of hypertension and type 2 diabetes (Writing Group Chinese Guidelines for the Management of Hypertension, 2010; Chinese Diabetes Society of Chinese Medical Association, 2014).

A total of 698 patients with type 2 diabetes with euthyroidism were collected and divided into five groups based on antihypertensive drugs, including patients without antihypertensive treatment as controls. The process of selection was illustrated in Figure 1.

Group NONE represent group without antihypertensive drug therapy. The medication included in other four groups were metoprolol ( $47.5 \mathrm{mg}$, qd), perindopril (4-8 mg, qd), telmisartan (40-80 mg, qd) and nifedipine (30 mg, qd, or $10-20 \mathrm{mg}$, bid) respectively.

Patients with history of serious cardiovascular events, stroke, chronic obstructive peripheral arteriopathy, et al. often treated with more than one antihypertensive medication. Patients treated with diuretics generally suffered from serious cardiovascular diseases in our hospital and treated with other antihypertensive drug at the same time, such as CCB and ACEI. These patients were not included.

The protocol was conducted in accordance with the Declaration of Helsinki and approved by the Beijing Anzhen Hospital Ethical Committee.

\section{Clinical Measurements and Biochemical Analysis}

The anthropometric indices (height and weight) were measured by professional medical workers using a standard protocol. Weight while wearing light clothing was measured to the nearest $0.1 \mathrm{~kg}$ and height to the nearest $1 \mathrm{~cm}$. Body mass index (BMI) was calculated as the body weight $(\mathrm{kg})$ divided by the square of the body height (m). Office BP was measured at the 


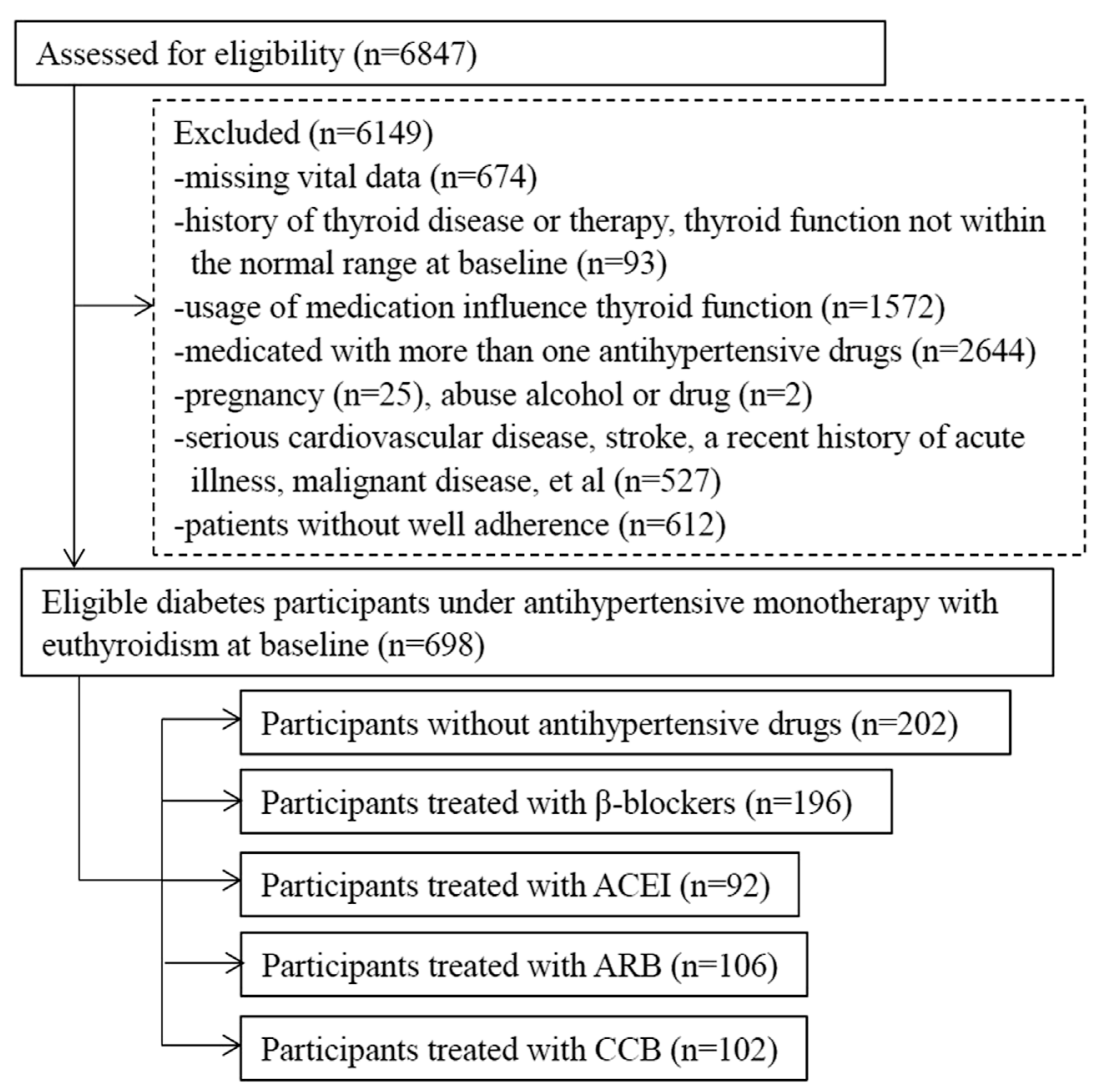

FIGURE 1 | The flowchart of selection.

right arm after a minimum of 5 min rest in the sitting position, using an automatic BP monitor (J30 [ $\pm 3 \mathrm{mmHg}$, Omron Healthcare Co., Ltd., Kyoto, Japan), and the average of two measurements were recorded. The sociodemographic characteristics, including age, sex, smoking status, alcohol consumption, individual history of diseases (including cardiovascular disease (CVD), hypertension, dyslipidemia, type 2 diabetes, complications of diabetes and hypertension, and thyroid disorders), and detailed drug treatment, were also collected from participants' medical records.

The venous blood samples were drawn between 6 after midnight (AM) to $8 \mathrm{AM}$ from overnight fasting at least $10 \mathrm{~h}$ subjects for the measurement of thyroid function, serum lipid and renal function. Serum concentrations of TSH (third-generation TSH assay; normal range: $0.49-4.91 \mathrm{mIU} / \mathrm{L}$ ), free triiodothyronine (fT3; normal range: 3.28-6.47 pmol/L), free thyroxine (fT4; normal range: 7.64-16.03 pmol/L), antithyroglobulin antibody (TgAb, normal range: $0-4.9 \mathrm{IU} / \mathrm{ml}$ ) and thyroperoxidases antibody (TPOAb, normal range: $0-9 \mathrm{IU} / \mathrm{ml}$ ) were measured using the ChemiLuminescence method by an automatic analyzer (UniCel DxI800 Access Immunoassay System, BECKMAN COULTER, United States) using reagents from BECKMAN COULTER, Inc. Serum total cholesterol (TC), LDL-C, HDL-C, and TGs were measured by colorimetry methods. Creatinine $(\mathrm{Cr})$ was measured by picric acid method. Fasting blood glucose (FBG) was measured using hexose kinase method. These measurements were performed on an automated instrument (Cobas 8000; Roche Diagnostics GmbH, Mannheim, Germany) using reagents from Roche diagnostics. Hemoglobin A1c (HbA1c) was measured using high performance liquid chromatography by $\mathrm{HbA1c}$ analyzer (Premier Hb9210, Primus Corporation, United States) using reagents from Trinity Biotech. The intraand interassay coefficients of variation were below $5 \%$ for the above parameters. Jostel's TSH index (TSHI) and thyroid's secretory capacity (SPINA-GT) were calculated using the equation described by Dietrich et al. (2016).

\section{Statistical Analysis}

The Shapiro-Wilk test was used to assess the distributional characteristic of variables. For continuous variables, data with normal distribution or approximate normal distribution were expressed with means and standard deviations (SD), or in the case of non-normal distribution, with medians and interquartile ranges (IQR). Categorical variables were recorded as absolute numbers with percentages.

Analysis of variance (ANOVA) followed by post hoc analysis of significance (Bonferroni's test) for continuous variables and Pearson's $\chi^{2}$ test for categorical variables were used to compare clinical data among different categories of antihypertensive therapy. A Welch test was used when the data were non- 
TABLE 1 | Clinical data of patients grouped according to their drug therapy at recruitment.

\begin{tabular}{|c|c|c|c|c|c|c|}
\hline & None & $\beta$-blockers & ACEI & ARB & CCB & $p$ value \\
\hline Patients (n) & 202 & 196 & 92 & 106 & 102 & \\
\hline Gender (n, \%) & $90(44.6 \%)$ & 98 (50.0\%) & $51(55.4 \%)$ & 39 (36.8\%) & $51(50.0 \%)$ & 0.071 \\
\hline Age (years) & $61.0 \pm 11.7$ & $63.5 \pm 9.9$ & $62.0 \pm 12.0$ & $63.2 \pm 13.0$ & $61.0 \pm 12.5$ & $0.135^{a}$ \\
\hline $\mathrm{BMI}\left(\mathrm{kg} / \mathrm{m}^{2}\right)$ & $25.710 \pm 3.373$ & $25.856 \pm 3.210$ & $26.418 \pm 3.037$ & $26.397 \pm 3.260$ & $26.310 \pm 3.155$ & 0.201 \\
\hline $\mathrm{SBP}(\mathrm{mmHg})$ & $128.376 \pm 13.355$ & $138.464 \pm 12.223$ & $136.728 \pm 11.731$ & $137.943 \pm 13.202$ & $136.069 \pm 13.434$ & $0.000^{b}$ \\
\hline $\mathrm{DBP}(\mathrm{mmHg})$ & $78.614 \pm 9.774$ & $83.709 \pm 9.317$ & $84.293 \pm 11.084$ & $85.113 \pm 10.794$ & $86.029 \pm 10.006$ & $0.000^{b}$ \\
\hline DM duration (years) & $4.0(6.0)$ & $3.0(7.0)$ & $5.0(9.0)$ & $5.0(9.0)$ & $5.0(8.3)$ & $0.087^{c}$ \\
\hline HT duration (years) & $4.0(5.0)$ & $6.0(7.0)$ & $6.0(13.8)$ & $7.0(10.3)$ & $6.0(8.0)$ & $0.000^{d}$ \\
\hline $\mathrm{TSH}(\mathrm{mlU} / \mathrm{L})$ & $2.304 \pm 0.902$ & $2.333 \pm 0.950$ & $2.332 \pm 1.023$ & $2.313 \pm 0.938$ & $2.394 \pm 0.931$ & 0.956 \\
\hline $\mathrm{fT}_{3}(\mathrm{pmol} / \mathrm{L})$ & $4.856 \pm 0.602$ & $4.874 \pm 0.654$ & $4.965 \pm 0.641$ & $4.782 \pm 0.585$ & $4.833 \pm 0.632$ & 0.341 \\
\hline $\mathrm{fT}_{4}(\mathrm{pmol} / \mathrm{L})$ & $11.053 \pm 1.781$ & $11.366 \pm 1.767$ & $11.286 \pm 1.655$ & $11.336 \pm 1.433$ & $11.198 \pm 1.798$ & 0.416 \\
\hline TPOAb/TgAb(+) (n, \%) & $18(8.9 \%)$ & $18(9.2 \%)$ & $8(8.7 \%)$ & $11(10.4 \%)$ & $9(8.8 \%)$ & 0.993 \\
\hline $\mathrm{TC}(\mathrm{mmol} / \mathrm{L})$ & $4.783 \pm 1.381$ & $4.544 \pm 1.215$ & $4.574 \pm 1.247$ & $4.509 \pm 0.989$ & $4.607 \pm 0.962$ & $0.304^{a}$ \\
\hline HDL-C (mmol/L) & $1.249 \pm 0.355$ & $1.245 \pm 0.347$ & $1.184 \pm 0.295$ & $1.272 \pm 0.342$ & $1.246 \pm 0.287$ & 0.447 \\
\hline LDL-C (mmol/L) & $2.791 \pm 1.004$ & $2.638 \pm 0.996$ & $2.617 \pm 1.054$ & $2.621 \pm 0.764$ & $2.680 \pm 0.833$ & $0.441^{a}$ \\
\hline TGs (mmol/L) & $1.897 \pm 1.914$ & $1.759 \pm 1.094$ & $2.077 \pm 1.636$ & $1.821 \pm 1.402$ & $1.929 \pm 1.556$ & 0.566 \\
\hline $\mathrm{FBG}(\mathrm{mmol} / \mathrm{L})$ & $7.563 \pm 2.416$ & $7.501 \pm 2.461$ & $8.004 \pm 2.491$ & $7.966 \pm 2.783$ & $7.836 \pm 3.050$ & 0.360 \\
\hline $\mathrm{HbA1c}(\%)$ & $6.839 \pm 1.453$ & $6.874 \pm 1.445$ & $7.034 \pm 1.426$ & $7.004 \pm 1.322$ & $7.145 \pm 1.773$ & 0.426 \\
\hline $\mathrm{Cr}(\mathrm{umol} / \mathrm{L})$ & $67.513 \pm 15.026$ & $69.899 \pm 14.578$ & $70.829 \pm 17.714$ & $68.724 \pm 15.038$ & $69.186 \pm 15.101$ & 0.414 \\
\hline eGFR (mL/min/1.73 m²) & $93.730 \pm 16.070$ & $89.811 \pm 14.208$ & $91.414 \pm 17.047$ & $90.004 \pm 16.490$ & $93.053 \pm 14.723$ & 0.080 \\
\hline Statin treatment $(n, \%)$ & $90(44.6 \%)$ & $96(49.0 \%)$ & 43 (46.7\%) & $48(45.3 \%)$ & 52 (51.0\%) & 0.815 \\
\hline Glucosidase inhibitors ( $n, \%)$ & 92 (45.5\%) & 94 (48.0\%) & 43 (46.7\%) & $58(54.7 \%)$ & 44 (43.1\%) & 0.503 \\
\hline Sulfonylureas ( $n, \%)$ & $60(29.7 \%)$ & 67 (34.2\%) & $29(31.5 \%)$ & 34 (32.1\%) & 38 (37.3\%) & 0.725 \\
\hline $\operatorname{TZD}(n, \%)$ & $45(22.3 \%)$ & $40(20.4 \%)$ & 15 (16.3\%) & 17 (16.0\%) & $18(17.6 \%)$ & 0.604 \\
\hline DPP-IV inhibitors (n, \%) & $53(26.2 \%)$ & 47 (24.0\%) & 19 (20.7\%) & 27 (25.5\%) & $25(24.5 \%)$ & 0.886 \\
\hline SGLT-2 inhibitors ( $n, \%)$ & $41(20.3 \%)$ & 36 (18.4\%) & 17 (18.5\%) & 28 (26.4\%) & 20 (19.6\%) & 0.539 \\
\hline Insulin (n, \%) & 78 (38.6\%) & 77 (39.3\%) & $43(46.7 \%)$ & 46 (43.4\%) & 41 (40.2\%) & 0.692 \\
\hline GLP-1 ra $(n, \%)$ & $42(20.8 \%)$ & $44(22.4 \%)$ & $13(14.1 \%)$ & $25(23.6 \%)$ & 20 (19.6\%) & 0.493 \\
\hline Retinopathy (n, \%) & 85 (42.1\%) & 95 (48.5\%) & 45 (48.9\%) & $53(50.0 \%)$ & 45 (44.1\%) & 0.583 \\
\hline Kidney disease (n, \%) & $85(42.1 \%)$ & $92(46.9 \%)$ & $46(50.0 \%)$ & $54(50.9 \%)$ & $50(49.0 \%)$ & 0.538 \\
\hline Peripheral neuropathy ( $n, \%)$ & $91(45.0 \%)$ & 96 (49.0\%) & $44(47.8 \%)$ & 55 (51.9\%) & 45 (44.1\%) & 0.746 \\
\hline Smokers $(n, \%)$ & $74(36.6 \%)$ & 67 (34.2\%) & $24(26.1 \%)$ & 30 (28.3\%) & 37 (36.3\%) & 0.304 \\
\hline Drinkers ( $n, \%)$ & 29 (14.4\%) & 30 (15.3\%) & 13 (14.1\%) & $18(17.0 \%)$ & $22(21.6 \%)$ & 0.535 \\
\hline
\end{tabular}

Data with normal distribution or approximate normal distribution were expressed as mean $\pm S D$. Data with non-normal distribution were expressed with medians and interquartile ranges (IQR). Categorical variables were recorded as absolute numbers with percentages. Retinopathy, kidney disease and peripheral neuropathy represent complications of diabetes and hypertension. TPOAb/TgAb(+) was defined as serum TPOAb/TgAb higher than their upper limit of reference ranges. $\beta$-blockers, selective $\beta_{1}$-adrenergic receptor blockers; $A C E l$, angiotensin-converting enzyme inhibitors; ARB, angiotensin receptor blockers; CCB, calcium channel blockers; BMI, body mass index; SBP, systolic blood pressure; DBP, diastolic blood pressure; DM, diabetes mellitus; HT, hypertension; TSH, thyroid-stimulating hormone; fT3, free triiodothyronine; fT4, free thyroxine; TPOAb, thyroperoxidases antibody; TgAb, antithyroglobulin antibody; TC, total cholesterol; HDL-C, high-density lipoprotein cholesterol; LDL-C, low-density lipoprotein cholesterol; TGs, triglycerides; FBG, fasting blood glucose; HbA1C, hemoglobin A1C; Cr, creatinine; eGFR, estimated glomerular filtration rate; TZD, thiazolidinediones; DPP-IV inhibitors, dipeptidyl peptidase-IV inhibitors; SGLT-2 inhibitors, sodium-dependent glucose transporters-2 inhibitors; GLP-1 ra, glucagon likepeptide-1 receptor agonists.

a Welch test is used for non-constant variance.

${ }^{b}$ Bonferroni's test revealed significant difference in SBP/DBP between patients without antihypertensive medication and other four groups, and no significance difference among the four groups with antihypertensive medication.

${ }^{c}$ Kruskal Wallis test is used for non-normal distribution variance.

${ }^{d}$ Kruskal Wallis test followed by Bonferroni's test for non-normal distribution variance revealed significant difference in HT duration between patients without antihypertensive medication and other four groups, and no significance difference among the four groups with antihypertensive medication.

constant variances in ANOVA. For data with non-normal distribution, a nonparametric test (Kruskal Wallis test followed by Bonferroni's test) was conducted. The measurements comparison between baseline and at the end of the study was performed by a paired student $t$-test, and Wilcoxon's Sign Rank Test was used for non-normal distribution data. D-values of data between the end of the study and baseline were calculated and the distribution was assessed. When comparing thyroid hormone level change under different antihypertensive medication, ANOVA and paired student $t$-test were both used and D-value was evaluated further.

Multiple logistic regression models with thyroid function levels at 1 year of medication as the dependent variable were used to identify the relationship between the thyroid function levels and the independent variables, including different antihypertensive therapy, gender, age, BMI, BP, smoking, alcohol consumption, diabetes/hypertension duration, HbAlc, serum lipid profile, estimated glomerular filtration rate (eGFR), thyroid autoimmunity, statin therapy, antidiabetes medicine, and complications of diabetes and hypertension. These were expressed as odds ratios (ORs) with 95\% confidence intervals (95\% CIs). The continuous variable of thyroid function level was transferred to a categorical variable. A dummy variable was set for transforming the categorical variable of antihypertensive medications, and the no antihypertensive treatment group was identified as control. 
TABLE 2 | Clinical data of patients at 1-year follow-up.

\begin{tabular}{|c|c|c|c|c|c|c|}
\hline & None & $\beta$-blockers & ACEI & ARB & CCB & $p$ value \\
\hline Patients $(n)$ & 202 & 196 & 92 & 106 & 102 & \\
\hline $\mathrm{BMl}\left(\mathrm{kg} / \mathrm{m}^{2}\right)$ & $25.825 \pm 3.220$ & $25.952 \pm 3.086$ & $26.168 \pm 2.896$ & $26.304 \pm 3.040$ & $26.206 \pm 2.986$ & 0.667 \\
\hline $\mathrm{SBP}(\mathrm{mmHg})$ & $128.980 \pm 10.665$ & $130.582 \pm 9.185$ & $129.565 \pm 9.321$ & $130.368 \pm 10.166$ & $128.235 \pm 9.412$ & 0.253 \\
\hline $\mathrm{DBP}(\mathrm{mmHg})$ & $80.252 \pm 6.453$ & $79.571 \pm 7.065$ & $81.315 \pm 6.709$ & $80.717 \pm 7.308$ & $81.235 \pm 6.774$ & 0.175 \\
\hline $\mathrm{TC}(\mathrm{mmol} / \mathrm{L})$ & $4.374 \pm 1.092$ & $4.214 \pm 1.059$ & $4.221 \pm 1.099$ & $4.394 \pm 0.829$ & $4.240 \pm 0.957$ & $0.356^{a}$ \\
\hline HDL-C (mmol/L) & $1.263 \pm 0.353$ & $1.211 \pm 0.354$ & $1.214 \pm 0.252$ & $1.264 \pm 0.286$ & $1.188 \pm 0.277$ & $0.170^{a}$ \\
\hline LDL-C (mmol/L) & $2.535 \pm 0.838$ & $2.389 \pm 0.818$ & $2.390 \pm 0.887$ & $2.516 \pm 0.642$ & $2.441 \pm 0.774$ & $0.344^{a}$ \\
\hline TGs (mmol/L) & $1.589 \pm 0.921$ & $1.787 \pm 1.381$ & $1.937 \pm 1.793$ & $1.692 \pm 1.178$ & $1.753 \pm 1.027$ & 0.233 \\
\hline FBG (mmol/L) & $6.996 \pm 1.681$ & $7.448 \pm 2.030$ & $7.415 \pm 1.665$ & $7.167 \pm 1.609$ & $7.097 \pm 1.933$ & 0.100 \\
\hline $\mathrm{HbA1c}(\%)$ & $6.620 \pm 0.999$ & $6.825 \pm 1.298$ & $6.952 \pm 1.047$ & $6.770 \pm 1.014$ & $6.811 \pm 1.247$ & 0.168 \\
\hline $\mathrm{Cr}(\mathrm{umol} / \mathrm{L})$ & $69.282 \pm 13.668$ & $71.643 \pm 14.042$ & $72.411 \pm 16.005$ & $70.545 \pm 13.514$ & $72.416 \pm 15.916$ & 0.266 \\
\hline eGFR $\left(\mathrm{mL} / \mathrm{min} / 1.73 \mathrm{~m}^{2}\right)$ & $91.596 \pm 16.017$ & $88.180 \pm 14.991$ & $89.787 \pm 16.441$ & $88.293 \pm 16.476$ & $89.485 \pm 16.376$ & 0.244 \\
\hline
\end{tabular}

Data with normal distribution or approximate normal distribution were expressed as mean $\pm S D$. $\beta$-blockers, selective $\beta_{1}$-adrenergic receptor blockers; ACEl, angiotensin-converting enzyme inhibitors; ARB, angiotensin receptor blockers; CCB, calcium channel blockers; BMl, body mass index; SBP, systolic blood pressure; DBP, diastolic blood pressure; TC, total cholesterol; HDL-C, high-density lipoprotein cholesterol; LDL-C, low-density lipoprotein cholesterol; TGs, triglycerides; FBG, fasting blood glucose; HbA1C, hemoglobin A1c; Cr, creatinine; eGFR, estimated glomerular filtration rate.

${ }^{a}$ Welch test is used for non-constant variance.

A $p$-value of less than 0.05 was regarded as statistically significant. We used Statistical Package for Social Sciences version 23.0 (SPSS Inc., ver.23, Chicago, IL, United States) for calculations and statistical analyses.

\section{RESULTS}

\section{Participant Characteristics}

Clinical data of patients subdivided into the five groups according to their drug therapy at recruitment are shown in Table 1. The differences in gender, age, BMI, cigarette smokers, and alcohol drinkers were not significant $(p>0.05)$ among the study groups. Serum TSH, $\mathrm{fT}_{3}, \mathrm{fT}_{4}$, thyroid antibodies, serum lipid profile, $\mathrm{FBG}$, $\mathrm{HbAlc}$, Cr, and eGFR were superimposable among the five groups $(p>0.05)$. All patients maintained diet-treatment and moderate activities. The percentages of antidiabetes and statins therapy, complications of diabetes and hypertension, duration of diabetes were comparable among the groups $(p>0.05)$. DBP and SBP was higher in antihypertensive treatment groups $(p<0.05)$, as well as the duration of hypertension was longer in antihypertensive treatment groups $(p<0.05)$, and no significant difference of DBP, SBP and duration of hypertension was found among the four antihypertensive treatment groups $(p>0.05)$.

The characteristics of subjects in the five groups at 1-year follow-up were provided in Table 2. There was no significant difference in index among the five groups, including SBP and DBP $(p>0.05)$. Positive TPOAb/TgAb did not change after treatment.

\section{Changes in Thyroid Function Among Participants Grouped According to Their Medication}

TSH levels increased only in patients undergoing metoprolol treatment and reached statistical significance (from $2.333 \pm$ 0.950 to $2.993 \pm 0.956 \mathrm{mIU} / \mathrm{L}, p<0.001)$. These data show that TSH serum levels were significantly higher in the group of patients treated with selective $\beta_{1}$-adrenergic receptor blockers compared with all other groups at the end of the study $(F=$ 16.176, $p<0.001)$. The values of TSH level did not differ among the other therapy groups (Table 3). The level of serum $\mathrm{fT}_{3}$ under the treatment of metoprolol decreased, but this was not statistically significant (from $4.874 \pm 0.654$ to $4.770 \pm$ $0.680 \mathrm{pmol} / \mathrm{L}, p=0.059$ ) (Table 4). Serum $\mathrm{fT}_{4}$ levels did not change significantly in any group from baseline to the end of the study (Table 5).

Then we proceeded to evaluate pituitary thyrotropic function indice TSHI, and the structure parameter of thyroid homeostasis SPINA-GT. The value of TSHI and SPINA-GT after and before $\beta_{1}$-blockers therapy were $(2.538 \pm 0.428$ vs $2.272 \pm 0.493)$ and (1.625 (0.559) pmol/s vs $1.861(0.964) \mathrm{pmol} / \mathrm{s})$, respectively. A significant increase and decrease was found in TSHI $(t=-9.119$, $p<0.001)$ and SPINA-GT $(z=8.125, p<0.001)$ after $\beta$-blocker therapy, respectively.

\section{Influencing Factors}

In order to conduct multiple logistic regression analysis, the continuous variable of D-value of TSH levels was transferred to a categorical variable. D-value was calculated by TSH level of after medication minus at baseline. We defined |D-value $\mid$ $\geq 0.5 \mathrm{mIU} / \mathrm{L}$ as a marked change. Pearson's $\chi^{2}$ test followed by post hoc analysis of significance (Bonferroni's test) revealed a statistical significance of proportion of $\mathrm{D}$-value with marked change under medication of selective $\beta_{1}$-adrenergic receptor blockers and other treatment $(p<0.001)$. Proportion of $\mathrm{D}$-value with marked change of other medications did not differ significantly (Table 6). This result was consistent with ANOVA analysis (Table 3) shown above.

The results of multiple logistic regression analysis with subjects with/without D-value of TSH with marked change as dependent variables, and antihypertensive drugs, gender, age, BMI, BP, smoking, alcohol consumption, diabetes/hypertension duration, serum lipid profile, HbAlc, eGFR, thyroid autoimmunity, statin therapy, antidiabetes medicine, and 
TABLE 3 | TSH levels at baseline and at the end of the study in each group (mIU/L).

\begin{tabular}{|c|c|c|c|c|c|}
\hline & Baseline & After medication & $t$ value & $p$ value & $\begin{array}{c}\text { D-value (after } \\
\text { medication-baseline) }\end{array}$ \\
\hline None & $2.304 \pm 0.902$ & $2.296 \pm 0.941$ & 0.195 & 0.846 & $-0.008 \pm 0.568$ \\
\hline$\beta$-blockers & $2.333 \pm 0.950$ & $2.993 \pm 0.956^{a}$ & -13.626 & 0.000 & $0.661 \pm 0.679^{b}$ \\
\hline ACEI & $2.332 \pm 1.023$ & $2.380 \pm 1.023$ & -0.812 & 0.419 & $0.048 \pm 0.565$ \\
\hline ARB & $2.313 \pm 0.938$ & $2.399 \pm 0.922$ & -1.625 & 0.107 & $0.086 \pm 0.542$ \\
\hline CCB & $2.394 \pm 0.931$ & $2.389 \pm 0.954$ & 0.094 & 0.925 & $-0.005 \pm 0.547$ \\
\hline$F$ value & 0.166 & 16.176 & & & 41.103 \\
\hline$p$ value & 0.956 & 0.000 & & & 0.000 \\
\hline
\end{tabular}

Data are presented as mean $\pm S D$. ACEl, angiotensin-converting enzyme inhibitors; ARB, angiotensin receptor blockers; CCB, calcium channel blockers; TSH, thyroid-stimulating hormone.

${ }^{a}$ Bonferroni's test revealed significant difference in TSH level between patients medication with $\beta$-blockers and other medication.

${ }^{b}$ Bonferroni's test revealed significant difference in D-value (after medication-baseline) between patients medication with $\beta$-blockers and other medication.

TABLE 4 | FT3 levels at baseline and at the end of the study in each group (pmol/L).

\begin{tabular}{|c|c|c|c|c|c|}
\hline & Baseline & After medication & $t$ value & $p$ value & $\begin{array}{c}\text { D-value (after } \\
\text { medication-baseline) }\end{array}$ \\
\hline None & $4.856 \pm 0.602$ & $4.881 \pm 0.571$ & -0.684 & 0.495 & $0.025 \pm 0.523$ \\
\hline$\beta$-blockers & $4.874 \pm 0.654$ & $4.770 \pm 0.680$ & 1.902 & 0.059 & $-0.104 \pm 0.766$ \\
\hline ACEI & $4.965 \pm 0.641$ & $4.913 \pm 0.603$ & 1.129 & 0.262 & $-0.051 \pm 0.435$ \\
\hline ARB & $4.782 \pm 0.585$ & $4.807 \pm 0.604$ & -0.356 & 0.723 & $0.024 \pm 0.701$ \\
\hline CCB & $4.833 \pm 0.632$ & $4.700 \pm 0.622$ & 1.699 & 0.092 & $-0.133 \pm 0.790$ \\
\hline$F$ value & 1.130 & 2.290 & & & 1.712 \\
\hline$p$ value & 0.341 & 0.058 & & & 0.145 \\
\hline
\end{tabular}

Data are presented as mean $\pm S D$. ACEl, angiotensin-converting enzyme inhibitors; ARB, angiotensin receptor blockers; CCB, calcium channel blockers; fT3, free triiodothyronine.

TABLE 5 | FT4 levels at baseline and at the end of the study in each group (pmol/L).

\begin{tabular}{|c|c|c|c|c|c|}
\hline & Baseline & After medication & $t$ value & $p$ value & $\begin{array}{c}\text { D-value (after } \\
\text { medication-baseline) }\end{array}$ \\
\hline None & $11.053 \pm 1.781$ & $11.307 \pm 1.653$ & -1.885 & 0.061 & $0.254 \pm 1.918$ \\
\hline$\beta$-blockers & $11.366 \pm 1.767$ & $11.166 \pm 1.719$ & 1.369 & 0.173 & $-0.201 \pm 2.053$ \\
\hline ACEI & $11.286 \pm 1.655$ & $11.579 \pm 1.811$ & -1.339 & 0.184 & $0.293 \pm 2.098$ \\
\hline ARB & $11.336 \pm 1.433$ & $11.159 \pm 1.544$ & 0.979 & 0.330 & $-0.177 \pm 1.865$ \\
\hline CCB & $11.198 \pm 1.798$ & $11.377 \pm 1.872$ & -0.865 & 0.389 & $0.180 \pm 2.100$ \\
\hline$F$ value & 0.983 & 1.142 & & & 2.085 \\
\hline$p$ value & 0.416 & 0.335 & & & 0.081 \\
\hline
\end{tabular}

Data are presented as mean $\pm S D$. ACEl, angiotensin-converting enzyme inhibitors; $A R B$, angiotensin receptor blockers; CCB, calcium channel blockers; $f$ 4, free thyroxine.

TABLE 6 | D-value (categorical variable transferred) of TSH level among each group.

\begin{tabular}{|c|c|c|c|c|c|c|}
\hline & None & $\beta$-blockers & ACEI & ARB & CСB & $F / p$ value \\
\hline Marked change & $63(31.2 \%)$ & $129(65.8 \%)^{a}$ & 34 (37.0\%) & 32 (30.2\%) & $28(27.5 \%)$ & $F=71.437$ \\
\hline Without marked change & $139(68.8 \%)$ & $67(34.2 \%)^{a}$ & $58(63.0 \%)$ & 74 (69.8\%) & 74 (72.5\%) & $p=0.000$ \\
\hline
\end{tabular}

Data are presented as absolute numbers with percentages. ACEl, angiotensin-converting enzyme inhibitors; ARB, angiotensin receptor blockers; CCB, calcium channel blockers; TSH, thyroid-stimulating hormone.

${ }^{a}$ Bonferroni's test revealed that the difference between prevalence of $D$-value with marked change under medication of selective $\beta$-blockers and other treatment was statistical significant.

complications of diabetes and hypertension at baseline as independent variables, are shown in Table 7. Compared with the non-antihypertensive treatment group, the effect on TSH levels under the usage of selective $\beta_{1}$-adrenergic receptor blockers was statistically significant $(O R=4.147$, 95\% CI 2.617-6.572, $p<0.001$ ) among the four antihypertensive drugs. Other confounding factors were not significant variables in this model. 
TABLE 7 | Multiple logistic regression analysis for prevalence of D-value of TSH with marked change as dependent variable in the whole group $(n=698)$.

\begin{tabular}{|c|c|c|c|c|}
\hline Variable & $B$ & $E X P(B)$ & $95 \% \mathrm{Cl}$ of $\operatorname{EXP}(B)$ & $p$ value \\
\hline Gender & 0.032 & 1.032 & $0.716-1.489$ & 0.865 \\
\hline Age & -0.018 & 0.982 & $0.963-1.001$ & 0.070 \\
\hline BMl & -0.028 & 0.973 & $0.924-1.024$ & 0.290 \\
\hline SBP & 0.007 & 1.007 & $0.993-1.020$ & 0.339 \\
\hline DBP & 0.001 & 1.001 & 0.983-1.019 & 0.935 \\
\hline DM duration & 0.013 & 1.013 & $0.979-1.048$ & 0.468 \\
\hline HT duration & 0.015 & 1.015 & $0.988-1.042$ & 0.289 \\
\hline $\mathrm{TC}$ & 0.072 & 1.074 & $0.752-1.536$ & 0.694 \\
\hline HDL-C & 0.033 & 1.033 & $0.511-2.090$ & 0.928 \\
\hline LDL-C & 0.037 & 1.038 & $0.704-1.530$ & 0.851 \\
\hline TGs & -0.047 & 0.954 & $0.794-1.147$ & 0.618 \\
\hline $\mathrm{HbA1c}$ & -0.125 & 0.883 & $0.778-1.001$ & 0.053 \\
\hline eGFR & -0.003 & 0.997 & $0.983-1.010$ & 0.637 \\
\hline TPOAb/TgAb(+) & -0.353 & 0.703 & $0.389-1.270$ & 0.243 \\
\hline Selective $\beta$-blockers & 1.422 & 4.147 & $2.617-6.572$ & 0.000 \\
\hline ACEI & 0.093 & 1.097 & $0.620-1.941$ & 0.750 \\
\hline ARB & -0.148 & 0.863 & $0.490-1.518$ & 0.609 \\
\hline CCB & -0.322 & 0.725 & $0.408-1.287$ & 0.272 \\
\hline Statins treatment & 0.311 & 1.364 & $0.971-1.916$ & 0.073 \\
\hline Glucosidase inhibitors & -0.211 & 0.810 & $0.563-1.165$ & 0.255 \\
\hline Sulfonylureas & 0.007 & 1.007 & $0.683-1.485$ & 0.972 \\
\hline Thiazolidinediones & -0.208 & 0.812 & $0.524-1.259$ & 0.352 \\
\hline DPP-IV inhibitors & -0.363 & 0.696 & $0.454-1.066$ & 0.096 \\
\hline SGLT-2 inhibitors & 0.042 & 1.043 & $0.687-1.582$ & 0.844 \\
\hline Insulin & 0.091 & 1.095 & $0.731-1.639$ & 0.659 \\
\hline GLP-1 receptor agonists & -0.332 & 0.718 & $0.459-1.122$ & 0.146 \\
\hline Retinopathy & -0.078 & 0.925 & $0.420-2.038$ & 0.846 \\
\hline Kidney disease & 0.273 & 1.314 & $0.579-2.984$ & 0.513 \\
\hline Peripheral neuropathy & -0.131 & 0.878 & $0.535-1.441$ & 0.606 \\
\hline Smokers & -0.299 & 0.741 & $0.501-1.097$ & 0.134 \\
\hline Drinkers & 0.246 & 1.279 & 0.779-2.099 & 0.330 \\
\hline
\end{tabular}

Retinopathy, kidney disease and peripheral neuropathy represent complications of diabetes and hypertension. TPOAb/TgAb(+) was defined as serum TPOAb/TgAb higher than their upper limit of reference ranges. TSH, thyroid-stimulating hormone; BMI, body mass index; SBP, systolic blood pressure; DBP, diastolic blood pressure; DM, diabetes mellitus; HT, hypertension; TC, total cholesterol; HDL-C, high-density lipoprotein cholesterol; LDL-C, low-density lipoprotein cholesterol; TGs, triglycerides; $H b A 1 C$, hemoglobin A1c; eGFR, estimated glomerular filtration rate; TPOAb, thyroperoxidases antibody; TgAb, antithyroglobulin antibody; ACEl, angiotensin-converting enzyme inhibitors; ARB, angiotensin receptor blockers; CCB, calcium channel blockers; DPP-IV inhibitors, dipeptidyl peptidase-IV inhibitors; SGLT-2 inhibitors, sodium-dependent glucose transporters-2 inhibitors; GLP-1 receptor agonists, glucagon likepeptide-1 receptor agonists.

\section{Changes in Thyroid Function in Participants Whose Selective $\beta_{1}$ - Adrenergic Receptor Blockers Were Discontinued After One Year's Medication}

In screening the computerized database, we found selective $\beta_{1^{-}}$ adrenergic receptor blockers were discontinued in 11 patients of group $\beta$-blockers after the elevation of TSH levels, for the following reasons: 1 ) heart rate $\leq 55$ beats per minute $(2 / 11)$; 2) medication treating hypertension was changed from $\beta$ blockers to $\operatorname{CCB}(3 / 11)$; 3) medication treating hypertension was changed from $\beta$-blockers to ACEI $(2 / 11) ; 4)$ exact reason not found (4/11). Seven men and four women were included. The doses of metoprolol medication of the 11 patients were $47.5 \mathrm{mg} / \mathrm{d}$. D-value of TSH level was calculated, and the distribution was assessed. A paired student $t$-test was conducted to evaluate TSH level changes. After 1 year's medication of selective $\beta$-blockers, TSH levels of the 11 patients were $(2.659 \pm 1.460) \mathrm{mIU} / \mathrm{L}$, a significant increase compared with the base level $(2.659 \pm 1.460$ vs. $1.814 \pm 1.293 \mathrm{mIU} / \mathrm{L}, \mathrm{D}$-value $=0.846$, 95\% CI $0.489-1.202$, $p=0.000)$. The period without metoprolol medication varied from three to 17 months. After withdrawal of metoprolol, TSH value dropped to the level close to baseline value at the beginning $(2.066 \pm 1.426$ vs, $1.814 \pm 1.293 \mathrm{mIU} / \mathrm{L}, \mathrm{D}$-value $=0.252,95 \% \mathrm{CI}$ -0.013 to $0.517, p=0.060)$.

\section{DISCUSSION}

The results of the retrospective study herein showed the TSHlifting effect of selective $\beta_{1}$-adrenergic receptor blockers, whereas no significant influence was found among other antihypertensive drugs. No matter whether we adjusted confounding factors or not, the association between $\beta$-blockers and thyroid functions existed. As far as we know, this was the first study to assess the association between thyroid function and antihypertensive drugs in patients with type 2 diabetes with euthyroidism.

$\beta$-adrenergic receptor blocking drugs have been widely used in clinical practice for more than 50 years (Frishman, 2008). Besides the $\beta$-blocking activity of this agent, another mechanism was the influence on the peripheral metabolism of circulating thyroid hormone. Studies consistently reported that $\beta$-adrenergic receptor blocking drugs decreases serum triiodothyronine $\left(\mathrm{T}_{3}\right)$ in patients with hyperthyroidism (Faber et al., 1979; Perret et al., 1984; Franklyn et al., 1985). However, similar serum $T_{3}$-lowering effects in patients with euthyroidism were inconsistent (Kristensen and Weeke, 1977; Perret et al., 1984; Franklyn et al., 1985; Wilkins et al., 1985; Kayser et al., 1991). There are also conflicting reports regarding the influence of $\beta$-adrenergic receptor blocking drugs on TSH levels in euthyroid patients (Kristensen and Weeke, 1977; Faber et al., 1979; Perret et al., 1984; Reeves et al., 1985; Kayser et al., 1991). Perret et al. (1984) reported that TSH concentrations remained stable under treatment with propranolol and acebutolol, which was in accordance with studies done by Faber et al. (1979) (propranolol), Kristensen and Weeke (1977) (propranolol), and Reeves et al. (1985) (propranolol and nadolol). But Kayser et al. have demonstrated that after a 1-week therapy of propranolol and atenolol, median serum TSH levels increased, whereas median serum TSH decreased compared with pretreatment values after 3 weeks of therapy. However, the increase of TSH levels under the treatment of metoprolol did not reach statistical significance (Kayser et al., 1991).

In this study, performed on larger sample size, we showed that treatment with metoprolol is associated with a significant rise in the serum levels of TSH in patients with euthyroid type 2 diabetes. The design of the current study was not aimed at a possible explanatory mechanism for the TSH-lifting effect of $\beta$-adrenergic receptor blocking drugs. The possible mechanisms from previous literatures were as followed. First, the regulatory $\beta_{1}$-adrenergic receptors normally mediated the inhibitory effect of epinephrine and norepinephrine on the TSH secretion from the pituitary 
gland (Kayser et al., 1991). Blocking of the regulatory $\beta_{1^{-}}$adrenergic receptors may cause an increase in serum TSH levels. But Melander et al. (1977) reported that adrenergic terminals had not been found in the hypothalamus or the pituitary of the mouse. In the current study, TSHI lifted under the treatment of $\beta_{1}$-adrenergic blockers might indicate an increase in TSH secretion. Our result required more verify. Second, the changes in the peripheral thyroxine $\left(\mathrm{T}_{4}\right)$ and $\mathrm{T}_{3}$ values could influence serum TSH levels in negative feedback mode. In Kuntz's literature, repeated proofs had indicated that thyroid gland function could be adjusted by sympathetic and parasympathetic nerves, and the nerve fibers terminate enter the thyroid gland might were distributed mainly to the thyroid follicles and the walls of the blood vessels (Kuntz, 1932). Melander et al. (1977) reported that the sympathetic nerve system could exert a direct, stimulatory effect on the secretion of the thyroid hormone, independently from TSH levels. However Hotta et al. (2017) discovered that the secretion of thyroid hormone increased during parasympathetic (superior laryngeal nerve) stimulation and decreased during sympathetic (cervical sympathetic trunk) stimulation, this result might associated with various nerve fibers were contained in superior laryngeal nerve, and further investigation was necessary. Our investigation showed SPINA-GT decreased by the sympathetic activity blocking effect under $\beta$-blockers treatment, which was consistent with Melander's animals study (Melander et al., 1977) and the mechanism of $\beta$-blockers, indicating the decreased secretion of thyroid hormone under $\beta$-blockers. Third, sympathetic nervous system may exert a stimulatory influence on the deiodination of thyroid hormone (Melander et al., 1977). Several studies supported that $\beta$ adrenergic receptor blockers decrease serum $\mathrm{T}_{3}$ due to inhibition of the conversion of $\mathrm{T}_{4}$ into $\mathrm{T}_{3}$ and provided evidence for an inhibitory influence of the type 1 or 2 deiodinase enzyme (Perrild et al., 1983; Wilkins et al., 1985; Wiersinga, 1991). This enzyme is responsible for the deiodinase from $T_{4}$ to $T_{3}$ (Chopra et al., 1978). The mechanism by which $\beta$-adrenergic receptor blockers exert this inhibition on the deiodinase enzyme is unclear. In the current study, a slight fall in serum $\mathrm{T}_{3}$ was found but was not statistically significant. But what should not be ignored was that the inhibition effect of the deiodinase enzyme was studied most on propranolol (unselective $\beta$-adrenergic receptor blockers). Our investigation was made on metoprolol, selective $\beta$-adrenergic receptor blockers, and the proof about inhibition effect by metoprolol was limited that was reported by Perrild' study (Perrild et al., 1983), which need more to confirm. As reported by Carr et al. (1988), even small alterations of thyroxine dosages given to patients with primary hypothyroidism could cause basal TSH values to change. Therefore, alterations of TSH level mediated by $\mathrm{T}_{4}$ or $\mathrm{T}_{3}$ value could not be excluded.

Above all, the effect that selective $\beta_{1}$-adrenergic receptor blockers may result in a rise in serum TSH levels is biologically reasonable, but studies to investigate the mechanisms are required.
A possible reason why no significant decrease in $\mathrm{T}_{3}$ and $\mathrm{T} 4$ level was found could be related to the medicine dose. In Wilkins's study (Wilkins et al., 1985), a significant fall of $\mathrm{T}_{3}$ level emerged with a higher propranolol dose. In this study, a relatively lower dose of metoprolol probably could not exert enough inhibitory influence to produce a significant fall of $\mathrm{T}_{3}$ and T4 level.

No significant variation was observed in serum TSH and thyroid hormone levels under the treatment of ACEI, ARB, and CCB. Renin-angiotensin-aldosterone system (RAAS) is activated by thyroid hormones in hyperthyroidism (Silverstein et al., 1983). But no association between ACEI and ARB treatment with thyroid function change was found with euthyroid ones in this study, in accordance with other clinical studies (Hume et al., 1990; Andjelkovic et al., 2016), conflicting with an animal study (Vranckx et al., 1995). The increased intracellular availability of $\mathrm{Ca}^{2+}$ plays an important role in the regulation and secretion of thyroid hormones (Lalau et al., 1987). Studies indicated that CCB could interfere with thyroid function through pituitary (Brabant et al., 1989; Roussel et al., 1995; Roussel et al., 1997), thyroid (Jangid, 1993; Pedemonte et al., 2005), and peripheral thyroid physiological process (Capen, 1994). However, the clinical studies on the effect of CCB on thyroid function were inconsistent (Isles et al., 1985; Haitas et al., 1986; Valensi et al., 1989). The probable mechanism there was no effect on thyroid function from CCB in the current study might was another $\mathrm{Ca}^{2+}$ pathway existing could not be prevented by CCB (Corda et al., 1985) and oral treatment (Semple et al., 1984).

Previous study showed that statins use was associated with lower TSH levels and the relationship was mediated by TC declines (Wang et al., 2021). Our investigation did not found this association might because TC reduction was not strong enough, which was partly consistent with Robert's study (Krysiak et al., 2019), or other hidden reason. Specially designed prospective study might be needed.

Decreased endothelial function, HDL-C level, and increased LDL-C level, TGs level, and BP were associated with higher TSH level even when TSH level was in normal range (Lekakis et al., 1997; Bakker et al., 2001; Asvold et al., 2007a; Asvold et al., 2007b), companied with a negative consequence on glucose metabolism by $\beta$-blockers treatment (Jacob et al., 1996), which remind clinical medical workers to pay attention to TSH-lifting effect in euthyroid diabetes patients treated with $\beta$-blockers. On the other hand, one of the most important therapeutic utility on non-cardiovascular diseases by $\beta$-blockers was on hyperthyroidism and thyroid storm. Except the blocking effect on sympathetic over-activity and of thyroid hormones in the cardiovascular system, the possible inhibitory effect on secretion of thyroid hormone by $\beta$-blockers may benefit the prognosis of hyperthyroidism and thyroid storm, which deserved more attention and required further confirmation.

This study has several limitations. First, this was a retrospective study. The most important criterion was to ensure the participants were under antihypertensive monotherapy throughout the follow-up. We tried to overcome this point by repeatedly confirming the medication with patients and excluded those without well adherence. However, we cannot 
be sure that there were no patients who did not follow the doctor's advice. Another disadvantage of this retrospective study was that the medication dosage could not be adjusted according to the study. Therefore, whether the dosage would influence the association could not be assessed. Also, causal relationship cannot be established due to the nature of an observational study. Thus, a further strictly designed prospective cohort study is needed to confirm our findings. Second, our study only considered subjects being treated with metoprolol, which questions whether our results could be applied to those being treated with other $\beta$-blockers. Third, although the intra- and interassay coefficients variation were below $5 \%$, and the blood sample was drawn between $6 \mathrm{AM}$ and $8 \mathrm{AM}$ after overnight fasting at least $10 \mathrm{~h}$, the influence of assay variation or diurnal variation to some extent might still exist. Finally, the patients were recruited from a single university hospital. Patients with serious cardiac events and other macrovascular complications were excluded because of more than one antihypertensive medicine treatment. Therefore, future multi-center prospective studies with participants suffering from macrovascular complications are needed to evidence the association.

\section{CONCLUSION}

In conclusion, these data reported the TSH-lifting effect of selective $\beta_{1^{-}}$adrenergic receptor blockers in diabetes with euthyroidism, which might result in higher CVD risks. Our result might also bring another proof about the ameliorated prognosis of hyperthyroidism and thyroid storm from the

\section{REFERENCES}

Alberti, K. G., and Zimmet, P. Z. (1999). Definition, Diagnosis and Classification of Diabetes Mellitus and its Complications Report of a WHO Consultation, Part 1: Diagnosis and Classification of Diabetes Mellitus. Diabet Med. 15, 539-553. doi:10.1002/(SICI)1096-9136(199807)

Andjelkovic, M., Jankovic, S., Mitrovic, M., Mladenovic, V., Nikolic, I., Zelen, I., et al. (2016). Effects of Cardiovascular Drugs on TSH Serum Levels in Patients on Replacement Therapy after Thyroidectomy. Int. J. Clin. Pharmacol. Ther. 54 (8), 628-633. doi:10.5414/CP202606

Asvold, B. O., Bjøro, T., Nilsen, T. I., and Vatten, L. J. (2007a). Association Between Blood Pressure and Serum Thyroid-Stimulating Hormone Concentration within the Reference Range: A Population-Based Study. J. Clin. Endocrinol. Metab. 92 (3), 841-845. doi:10.1210/jc.2006-2208

Asvold, B. O., Vatten, L. J., Nilsen, T. I., and Bjøro, T. (2007b). The Association Between TSH Within the Reference Range and Serum Lipid Concentrations in a Population-Based Study. The HUNT Study. Eur. J. Endocrinol. 156 (2), 181-186. doi:10.1530/eje.1.02333

Bakker, S. J., ter Maaten, J. C., Popp-Snijders, C., Slaets, J. P., Heine, R. J., and Gans, R. O. (2001). The Relationship Between Thyrotropin and Low Density Lipoprotein Cholesterol is Modified by Insulin Sensitivity in Healthy Euthyroid Subjects. J. Clin. Endocrinol. Metab. 86 (3), 1206-1211. doi:10.1210/jcem.86.3.7324

Bakris, G. L., Fonseca, V., Katholi, R. E., McGill, J. B., Messerli, F. H., Phillips, R. A., et al. (2004). Metabolic Effects of Carvedilol vs Metoprolol in Patients with Type 2 Diabetes Mellitus and Hypertension: a Randomized Controlled Trial. JAMA 292 (18), 2227-2236. doi:10.1001/jama.292.18.2227

Brabant, G., Ocran, K., Ranft, U., von zur Mühlen, A., and Hesch, R. D. (1989). Physiological Regulation of Thyrotropin. Biochimie 71 (2), 293-301. doi:10.1016/0300-9084(89)90066-7 possible inhibitory effect on secretion of thyroid hormone by $\beta_{1^{-}}$adrenergic receptor blockers. Further prospective studies conducted on a larger series of patients are required to confirm the association.

\section{DATA AVAILABILITY STATEMENT}

The original contributions presented in the study are included in the article/supplementary material, further inquiries can be directed to the corresponding author.

\section{ETHICS STATEMENT}

The studies involving human participants were reviewed and approved by Beijing Anzhen Hospital, Capital Medical University ethics committee (2022021X). The patients/ participants provided their written informed consent to participate in this study.

\section{AUTHOR CONTRIBUTIONS}

Conception and design of the research: LY, YZ, HT. Acquisition of data: LY, XS. Analysis and interpretation of the data: LY, YZ, HT. Statistical analysis: LY, XS. Writing of the manuscript: LY, XS. Critical revision of the manuscript for intellectual content: YZ, HT. All authors read and approved the final draft.

Capen, C. C. (1994). Mechanisms of Chemical Injury of Thyroid Gland. Prog. Clin. Biol. Res. 387, 173-191.

Cappelli, C., Rotondi, M., Pirola, I., Agosti, B., Formenti, A., Zarra, E., et al. (2012). Thyreotropin Levels in Diabetic Patients on Metformin Treatment. Eur. J. Endocrinol. 167 (2), 261-265. doi:10.1530/EJE-12-0225

Carr, D., McLeod, D. T., Parry, G., and Thornes, H. M. (1988). Fine Adjustment of Thyroxine Replacement Dosage: Comparison of the Thyrotrophin Releasing Hormone Test Using a Sensitive Thyrotrophin Assay with Measurement of Free Thyroid Hormones and Clinical Assessment. Clin. Endocrinol. 28 (3), 325-333. doi:10.1111/j.1365-2265.1988.tb01219.x

Celik, T., Iyisoy, A., Kursaklioglu, H., Kardesoglu, E., Kilic, S., Turhan, H., et al. (2006). Comparative Effects of Nebivolol and Metoprolol on Oxidative Stress, Insulin Resistance, Plasma Adiponectin and Soluble P-Selectin Levels in Hypertensive Patients. J. Hypertens. 24 (3), 591-596. doi:10.1097/ 01.hjh.0000209993.26057.de

Chinese Diabetes Society of Chinese Medical Association (2014). Chinese Guidelines for the Management of Type 2 Diabetes (2013). Chin. J. Diabetes 6 (7), 447-498. doi:10.3760/cma.j.issn.16745809.2014.07.004

Chopra, I. J., Solomon, D. H., Chopra, U., Wu, S. Y., Fisher, D. A., and Nakamura, Y. (1978). Pathways of Metabolism of Thyroid Hormones. Recent Prog. Horm. Res. 34, 521-567. doi:10.1016/b978-0-12-571134-0.50018-1

Corda, D., Marcocci, C., Kohn, L. D., Axelrod, J., and Luini, A. (1985). Association of the Changes in Cytosolic Ca2+ and Iodide Efflux Induced by Thyrotropin and by the Stimulation of Alpha 1-adrenergic Receptors in Cultured Rat Thyroid Cells. J. Biol. Chem. 260 (16), 9230-9236. doi:10.1016/s00219258(17)39357-2

Dietrich, J. W., Landgrafe-MendeKlein, G., Wiora, E., Chatzitomaris, A., Klein, H. H., Midgley, J. E. M., et al. (2016). Calculated Parameters of Thyroid 
Homeostasis: Emerging Tools for Differential Diagnosis and Clinical Research. Front. Endocrinol. 7, 57. doi:10.3389/fendo.2016.00057

Eidne, K. A., Zabavnik, J., Allan, W. T., Trewavas, A. J., Read, N. D., and Anderson, L. (1994). Calcium Waves and Dynamics Visualized by Confocal Microscopy in Xenopus Oocytes Expressing Cloned TRH Receptors. J. Neuroendocrinol 6 (2), 173-178. doi:10.1111/j.13652826.1994.tb00569.x

Faber, J., Friis, T., Kirkegaard, C., Lumholtz, I. B., Hansen, J. M., SiersbaekNielsen, K., et al. (1979). Serum T4, T3 and Reverse T3 During Treatment with Propranolol in Hyperthyroidism, L-T4 Treated Myxedema and in Normal Man. Horm. Metab. Res. 11 (1), 34-36. doi:10.1055/s-00281092678

Franklyn, J. A., Wilkins, M. R., Wilkinson, R., Ramsden, D. B., and Sheppard, M. C. (1985). The Effect of Propranolol on Circulating Thyroid Hormone Measurements in Thyrotoxic and Euthyroid Subjects. Acta Endocrinol. 108 (3), 351-355. doi:10.1530/acta.0.1080351

Frishman, W. H. (2008). Beta-Adrenergic Blockers: A 50-Year Historical Perspective. Am. J. Ther. 15 (6), 565-576. doi:10.1097/ MJT.0b013e318188bdca

Haitas, B., Joffe, B. I., Edelstein, D., Panz, V., Lamprey, J. M., and Seftel, H. C. (1986). Calcium Antagonists (Nifedipine and Nimodipine) and Pituitary Responses to Thyrotropin Releasing Hormone Stimulation. J. Cardiovasc. Pharmacol. 8 (6), 1284-1286. doi:10.1097/00005344198611000-00028

Hotta, H., Onda, A., Suzuki, H., Milliken, P., and Sridhar, A. (2017). Modulation of Calcitonin, Parathyroid Hormone, and Thyroid Hormone Secretion by Electrical Stimulation of Sympathetic and Parasympathetic Nerves in Anesthetized Rats. Front. Neurosci. 11 (30), 375. doi:10.3389/fnins.2017.00375

Hume, A. L., Jack, B. W., and Levinson, P. (1990). Severe Hyponatremia: An Association with Lisinopril? DICP 24 (12), 1169-1172. doi:10.1177/ 106002809002401204

Isles, T. E., Baty, J., and Dow, R. J. (1985). The Effect of Nicardipine on Pituitary Hormone Release in normal Volunteers. Br. J. Clin. Pharmacol. 20 (Suppl. 1), 84S-87S. doi:10.1111/j.1365-2125.1985.tb05147.x

Jacob, S., Rett, K., Wicklmayr, M., Agrawal, B., Augustin, H. J., and Dietze, G. J. (1996). Differential Effect of Chronic Treatment with Two Beta-Blocking Agents on Insulin Sensitivity: the Carvedilol-Metoprolol Study. J. Hypertens. 14 (4), 489-494. doi:10.1097/00004872-199611000-0002310.1097/00004872199604000-00012

Jangid, D. R. (1993). Nifedipine Induced Modulations of Intra-thyroidal RadioIodine Turnover Kinetics in Euthyroid and Thyrotoxic Patients. J. Assoc. Physicians India 41 (10), 655-656, 661.

Kayser, L., Perrild, H., Feldt-Rasmussen, U., Hegedüs, L., Skovsted, L., and Hansen, J. E. (1991). The Thyroid Function and Size in Healthy Man during 3 Weeks Treatment with Beta-Adrenoceptor-Antagonists. Horm. Metab. Res. 23 (1), 35-37. doi:10.1055/s-2007-1003605

Klein, I., and Ojamaa, K. (2001). Thyroid Hormone and the Cardiovascular System. N. Engl. J. Med. 344 (7), 501-509. doi:10.1056/ NEJM200102153440707

Kristensen, B. O., and Weeke, J. (1977). Propranolol-Induced Increments in Total and Free Serum Thyroxine in Patients with Essential Hypertension. Clin. Pharmacol. Ther. 22 (6), 864-867. doi:10.1002/cpt1977226864

Krysiak, R., Szkróbka, W., and Okopień, B. (2019). Atorvastatin Potentiates the Effect of Selenomethionine on Thyroid Autoimmunity in Euthyroid Women with Hashimoto's Thyroiditis. Curr. Med. Res. Opin. 35 (4), 675-681. doi:10.1080/03007995.2018.1541314

Kuntz, A. (1932). Functional Interrelationships of the Thyroid Gland and the Autonomic Nervous System. Radiology 18(3), 532-535. doi:10.1148/ 18.3.532

Lalau, J. D., Miteljan, E., Arlot, S., and Quichaud, J. (1987). Effect of Three Days Nifedipine Administration on Thyroid Response to TSH. Horm. Metab. Res. 19 (1), 40. doi:10.1055/s-2007-1011732

Lekakis, J., Papamichael, C., Alevizaki, M., Piperingos, G., Marafelia, P., Mantzos, J., et al. (1997). Flow-mediated, Endotheliumdependent Vasodilation Is Impaired in Subjects with Hypothyroidism, Borderline Hypothyroidism, and High-normal
Serum Thyrotropin (TSH) Values. Thyroid 7 (3), 411-414. doi:10.1089/thy.1997.7.411

Melander, A., Westgren, U., Ericson, L. E., and Sundler, F. (1977). Influence of the Sympathetic Nervous System on the Secretion and Metabolism of Thyroid Hormone. Endocrinology 101 (4), 1228-1237. doi:10.1210/endo101-4-1228

Mullur, R., Liu, Y. Y., and Brent, G. A. (2014). Thyroid Hormone Regulation of Metabolism. Physiol. Rev. 94 (2), 355-382. doi:10.1152/ physrev.00030.2013

Pedemonte, N., Diena, T., Caci, E., Nieddu, E., Mazzei, M., Ravazzolo, R., et al. (2005). Antihypertensive 1,4-dihydropyridines as Correctors of the Cystic Fibrosis Transmembrane Conductance Regulator Channel Gating Defect Caused by Cystic Fibrosis Mutations. Mol. Pharmacol. 68 (6), 1736-1746. doi:10.1124/mol.105.015149

Perret, G., Hugues, J. N., Modigliani, E., and Sebaoun, J. (1984). Compared Effect of Propranolol and Acebutolol on Serum Thyroid Hormones' Levels in Euthyroid and Hyperthyroid Patients: a Randomized Study. Int. J. Clin. Pharmacol. Ther. Toxicol. 22 (7), 360-364.

Perrild, H., Hansen, J. M., Skovsted, L., and Christensen, L. K. (1983). Different Effects of Propranolol, Alprenolol, Sotalol, Atenolol and Metoprolol on Serum T3 and Serum rT3 in Hyperthyroidism. Clin. Endocrinol. 18 (2), 139-142. doi:10.1111/j.1365-2265.1983.tb03196.x

Punthakee, Z., Werstuck, G. H., and Gerstein, H. C. (2007). Diabetes and Cardiovascular Disease: Explaining the Relationship. Rev. Cardiovasc. Med. 8 (3), 145-153.

Reeves, R. A., From, G. L., Paul, W., and Leenen, F. H. (1985). Nadolol, Propranolol, and Thyroid Hormones: Evidence for a Membrane-Stabilizing Action of Propranolol. Clin. Pharmacol. Ther. 37 (2), 157-161. doi:10.1038/ clpt.1985.28

Rizos, E., Bairaktari, E., Kostoula, A., Hasiotis, G., Achimastos, A., Ganotakis, E., et al. (2003). The Combination of Nebivolol Plus Pravastatin Is Associated with a More Beneficial Metabolic Profile Compared to that of Atenolol Plus Pravastatin in Hypertensive Patients with Dyslipidemia: A Pilot Study. J. Cardiovasc. Pharmacol. Ther. 8 (2), 127-134. doi:10.1177/ 107424840300800206

Roussel, J. P., Grazzini, E., and Astier, H. (1997). Somatostatin Blocks the Potentiation of TRH-Induced TSH Secretion from Perifused Pituitary Fragments and the Change in Intracellular Calcium Concentrations from Dispersed Pituitary Cells Elicited by Prepro-TRH (PS4) or by Triiodothyronine. J. Mol. Endocrinol. 19 (1), 87-97. doi:10.1677/ jme.0.0190087

Roussel, J. P., Grazzini, E., Zumbihl, R., Rodriguez, E., and Astier, H. (1995). Triiodo-L-thyronine Enhances TRH-Induced TSH Release from Perifused Rat Pituitaries and Intracellular Ca2+ Levels from Dispersed Pituitary Cells. Eur. J. Pharmacol. 289 (2), 205-215. doi:10.1016/0922-4106(95) 90096-9

Semple, C. G., Thomson, J. A., Beastall, G. H., and Lorimer, A. R. (1984). Calcium Antagonists and Endocrine Status: Lack of Effect of Oral Verapamil on Pituitary-Testicular and Pituitary-Thyroid Function. Br. J. Clin. Pharmacol. 17 (2), 179-182. doi:10.1111/j.13652125.1984.tb02336.x

Silverstein, E., Schussler, G. C., and Friedland, J. (1983). Elevated Serum Angiotensin-Converting Enzyme in Hyperthyroidism. Am. J. Med. 75 (2), 233-236. doi:10.1016/0002-9343(83)91198-1

Valensi, P., PerretR Vassy, G., Vassy, R., Uzzan, B., Nicolas, P., and Attali, J. R. (1989). Effect of Nifedipine on Thyrotropin, Prolactin, and Thyroid Hormone Release in Man: A Placebo-Controlled Study. Fundam. Clin. Pharmacol. 3 (1), 59-66. doi:10.1111/j.1472-8206.1989.tb00031.x

Vranckx, R., Savu, L., Lambert, N., de Conchard, G. V., Grosse, R., Mourey, M. S., et al. (1995). Plasma Proteins as Biomarkers of the Aging Process. Am. J. Physiol. 268 (2 Pt 2), R536-R548. doi:10.1152/ ajpregu.1995.268.2.R536

Wang, Y., Li, Q., Yuan, Z., Ma, S., Shao, S., Wu, Y., et al. (2021). Statin Use and Benefits of Thyroid Function: A Retrospective Cohort Study. Front. Endocrinol. 12, 578909. doi:10.3389/fendo.2021.578909

Wiersinga, W. M. (1991). Propranolol and Thyroid Hormone Metabolism. Thyroid 1 (3), 273-277. doi:10.1089/thy.1991.1.273 
Wilkins, M. R., Franklyn, J. A., Woods, K. L., and Kendall, M. J. (1985). Effect of Propranolol on Thyroid Homeostasis of Healthy Volunteers. Postgrad. Med. J. 61 (715), 391-394. doi:10.1136/pgmj.61.715.391

Writing Group of Chinese Guidelines for the Management of Hypertension (2010). 2010 Chinese Guidelines for the Management of Hypertension. Chin. J. Front. Med. Sci. 3 (5), 42-93.

Zhang, H., Xiang, H., and Yang, Y. (2009). Causes of Death in Inpatients with Diabetes in 15 Provinces from 1991 to 2005. Chin. J. Diabetes 17 (1), 6-8. doi:10.3969/j.issn.1006-6187.2009.01.003

Conflict of Interest: The authors declare that the research was conducted in the absence of any commercial or financial relationships that could be construed as a potential conflict of interest.
Publisher's Note: All claims expressed in this article are solely those of the authors and do not necessarily represent those of their affiliated organizations, or those of the publisher, the editors and the reviewers. Any product that may be evaluated in this article, or claim that may be made by its manufacturer, is not guaranteed or endorsed by the publisher.

Copyright (c) 2022 Yang, Sun, Zhao and Tao. This is an open-access article distributed under the terms of the Creative Commons Attribution License (CC $B Y)$. The use, distribution or reproduction in other forums is permitted, provided the original author(s) and the copyright owner(s) are credited and that the original publication in this journal is cited, in accordance with accepted academic practice. No use, distribution or reproduction is permitted which does not comply with these terms. 


\section{GLOSSARY}

95\% CIs 95\% confidence intervals

ACEI angiotensin-converting enzyme inhibitors

AM after midnight

ANOVA analysis of variance

ARB angiotensin receptor blockers

BMI body mass index

BP blood pressure

CCB calcium channel blockers

$\mathrm{Cr}$ creatinine

CVD cardiovascular disease

DBP diastolic blood pressure

DPP-IV inhibitors dipeptidyl peptidase-IV inhibitors

eGFR estimated glomerular filtration rate

fT3 free triiodothyronine

fT4 free thyroxine

FBG fasting blood glucose

GLP-1 ra glucagon likepeptide-1 receptor agonists

HbA1c Hemoglobin A1c
HDL-C high-density lipoprotein cholesterol

HT hypertension

IQR interquartile ranges

LDL-C low-density lipoprotein cholesterol

ORs odds ratios

RAAS renin-angiotensin-aldosterone system

SBP systolic blood pressure

SD standard deviations

SGLT-2 inhibitors sodium-dependent glucose transporters-2 inhibitors SPINA-GT thyroid's secretory capacity

T3 triiodothyronine

T4 thyroxine

TC total cholesterol

TgAb antithyroglobulin antibody

TGs triglycerides

TPOAb thyroperoxidases antibody

TSH thyroid-stimulating hormone

TSHI Jostel's TSH index

TZD thiazolidinediones

WHO World Health Organization 